\title{
Discontinuation of teriflunomide and dimethyl fumarate in a large Italian multicentre population: a 24-month real-world experience
}

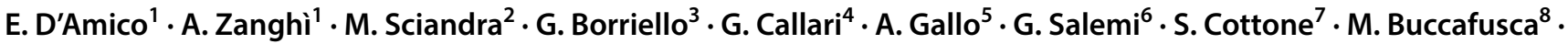

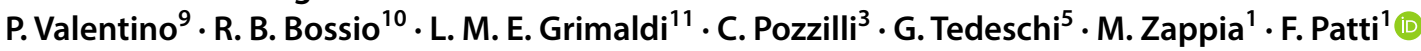

Received: 20 September 2018 / Revised: 7 November 2018 / Accepted: 26 November 2018 / Published online: 4 December 2018

(c) Springer-Verlag GmbH Germany, part of Springer Nature 2018

\begin{abstract}
Background Teriflunomide (TRF) and Dimethyl fumarate (DMF) are licensed drugs for relapsing-remitting Multiple Sclerosis (RRMS).

Objectives We aimed to compare the rate and the time to discontinuation among persons with RRMS (pwRRMS), newly treated with TRF and DMF.

Materials and methods A retrospective study on prospectively collected data was performed in nine tertiary MS centers, in Italy. The 24-month discontinuation rate in the two cohorts was the primary study outcome. We also assessed the time to discontinuation and reasons of therapy withdrawn. Discontinuation of TRF and DMF was defined as a gap of treatment $\geq 60$ days.

Results A cohort of 903 pwRRMS (316 on TRF and 587 on DMF) was analyzed. During 24 months of follow-up, pwRRMS on TRF and DMF showed similar discontinuation rates. The analysis of predictors with Cox regression model showed differences between the two groups ( $p$ for log-rank test $=0.007$ ); male gender [HR $2.21(1.00-4.90) ; p=0.01$ ] and the number of previous switches [HR $1.47(1.16-1.86) ; p=0.01]$ were associated with higher hazard of discontinuation in the DMF group. Conclusions In a real-world setting, pwRRMS on TRF and DMF had similar discontinuation rates over 24 months. Male pwRRMS on DMF with a previous history of therapeutic failure are at more risk of discontinuation therapy.
\end{abstract}

Keywords Dimethyl fumarate $\cdot$ Teriflunomide $\cdot$ Discontinuation rate $\cdot$ Real-life $\cdot$ Multiple sclerosis

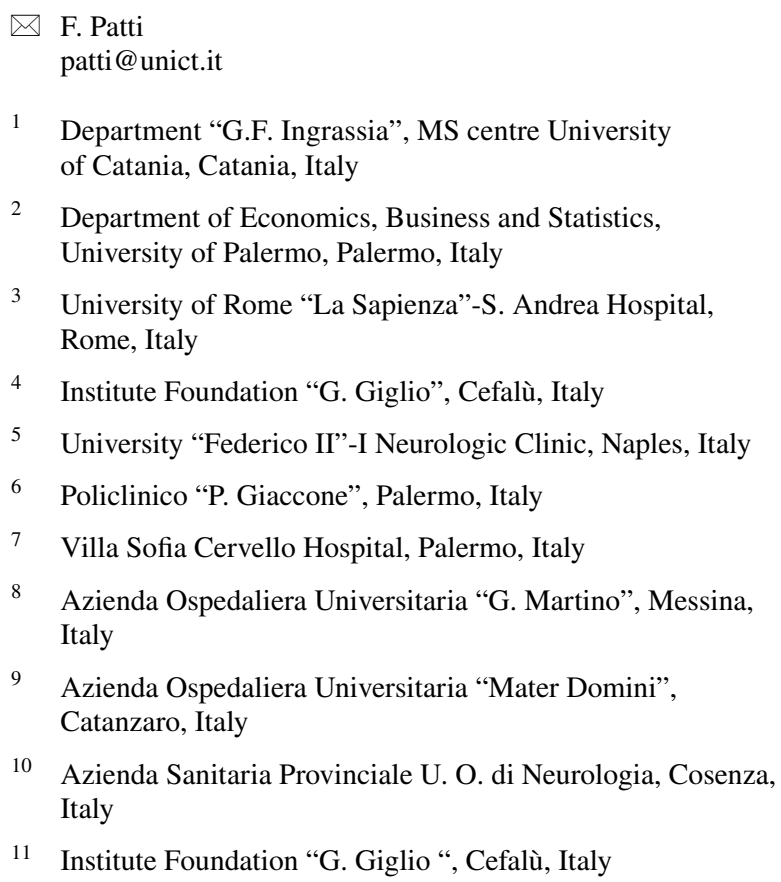

\section{Introduction}

The current therapeutic approach for the relapsing remitting forms of multiple sclerosis (RRMS) is based on the early start of treatment with one of the licensed disease modifying therapies (DMTs) with the possibility to switch to another DMT in case of failure of efficacy or for raising safety alert(s) [1,2]. In the past decade, oral first-line agents have been approved, broadening a new era of personalized therapy $[3,4]$. For medications to be most effective, patients must take them as prescribed by their neurologists and for the prescribed duration of treatment (that is to be persistent on therapy). Real-world studies have shown that not taking DMTs as prescribed can lead to greater risk for negative clinical and economic outcomes [5].

Studies comparing the discontinuation rates and time to discontinuation among oral drugs in RRMS are increasing [6-11], and a recent Italian study tracked and evaluated the post-market DMF profile in real-world setting [12]. 
We compared the discontinuation rates in two large groups of persons with RRMS (pwRRMS) treated with TRF and DMF, using a large Italian multicentre cohort. Moreover, we described the time to discontinuation and the reasons of DMTs withdrawn.

Moreover, we assessed the baseline factors that could predict the treatment discontinuation.

\section{Methods}

The inclusion criteria were: (1) age $\geq 18$; (2) a diagnosis of RRMS per 2010 McDonald criteria [13]; (3) beginning of the study therapies (TRF and DMF) in the index window (January 1, 2015-March 1, 2016); (4) to be not enrolled in randomized clinical trials.

The demographic, clinical, and brain magnetic resonance imaging (MRI) data were recorded retrospectively (up to 12 months) before the start of treatment with TRF or DMF (the index date) and prospectively (up to 24 months or until the last available visit of follow-up) from the index date.

The enrolled pwRRMS population is the same as described in a previous paper, which was performed with a propensity score matched population of 234 pwRRMS for each cohort [14].

The index drugs were prescribed at the following dosages: TRF at $14 \mathrm{mg}$ daily; DMF $120 \mathrm{mg}$ daily for 7 days followed by $240 \mathrm{mg}$ twice daily.

The data entry portal was iMED( $\odot$ software and we followed a rigorous quality assurance procedure with a doubleentered data system, which is defined as the definitive gold standard of clinical practice for data from collected paper forms. The research has been conducted according to the World Medical Association Declaration of Helsinki. The manuscript is conformed to ICMJE Recommendations for the Conduct, Reporting, Editing, and Publication of Scholarly Work in Medical Journals. Written informed consents were collected from all enrolled patients. This study was approved by the Policlinico-Vittorio Emanuele (Catania, Italy) Ethics Committee (no. 177/2017/PO).

No financial support for design, data collection, analysis, interpretation, or writing was received.

\section{Outcomes}

The 24-month discontinuation rate was the primary study outcome. We also assessed the time to discontinuation and the reasons of therapy withdrawn. Moreover, we explored the baseline demographic, clinical and radiological factors associated with therapy discontinuation.

Discontinuation of TRF or DMF was defined as a gap of treatment $\geq 60$ days.
Time to discontinuation (in months) was measured as time between the index date and the end of supply of the TRF or DMF prescriptions dispensed.

Adverse events (AEs) were collected, according to the European Medical Agencies (EMA) definitions [15].

\section{Statistical analysis}

Univariate comparisons were made through the ANOVA, Kruskal-Wallis or Chi-squared test. Persistence at 12 and 24 months was compared using a $\chi^{2}$ test. The Kaplan-Meier survival curves analysis and log-rank test were used to analyze the time to drug discontinuation. In our sensitivity analyses, we changed the permissible treatment gap period to 30 and 180 days.

A Cox proportional hazards model adjusting for differences among the treatment cohorts was used to assess the risk of discontinuation. Covariates in the analyses included gender, EDSS score during baseline period (in the year previous index date; median), previous MRI activity (in the year previous index date; number of new $\mathrm{T} 2$ or gadolinium lesions), MS relapse during the baseline period (in the year previous index date; total number), previous oral and injectable/infused DMT use (yes/no), and previous DMT switches (total number of switches before index date). Given the potential for correlation among the two-time varying covariates age and disease duration, we included in the linear predictor a new variable called conditional duration age (CDA) defined as the raw residuals of the linear model between $Y=$ disease duration and $X=$ age. CDA coefficient represents the mean of the pwRRMS' distances between observed durations and the duration mean predicted by taking into account patients' ages.

Results were considered significant for $p$ values $<0.05$ (two sided). SPSS version 21 was used (IBM SPSS Statistics 21, IBM, Armonk, NY, USA).

\section{Results}

From a total sample of 6480 pwRRMS from nine Italian MS centers, 903 were considered eligible for analyses. Of those, 316 pwRRMS were on TRF and 587 on DMF and met the inclusion criteria to be included in the analyses (Fig. 1).

Table 1 shows the pwRRMS characteristics at baseline. PwRRMS on TRF were older, with a longer disease duration, higher median EDSS score ( $p<0.05$; for all). Moreover, a higher number of pwRRMS on TRF was naïve to treatment than pwRRMS on DMF $(p<0.05)$.

Using a treatment gap of 60 days, we identified a total of 81 pwRRMS (8.9\%) who discontinued their treatment within the entire follow-up period. 
Fig. 1 Flow chart of the study. $D M F$ dimethyl fumarate, $p w R$ $R M S$ patients with relapsing remitting multiple sclerosis, $T R F$ teriflunomide

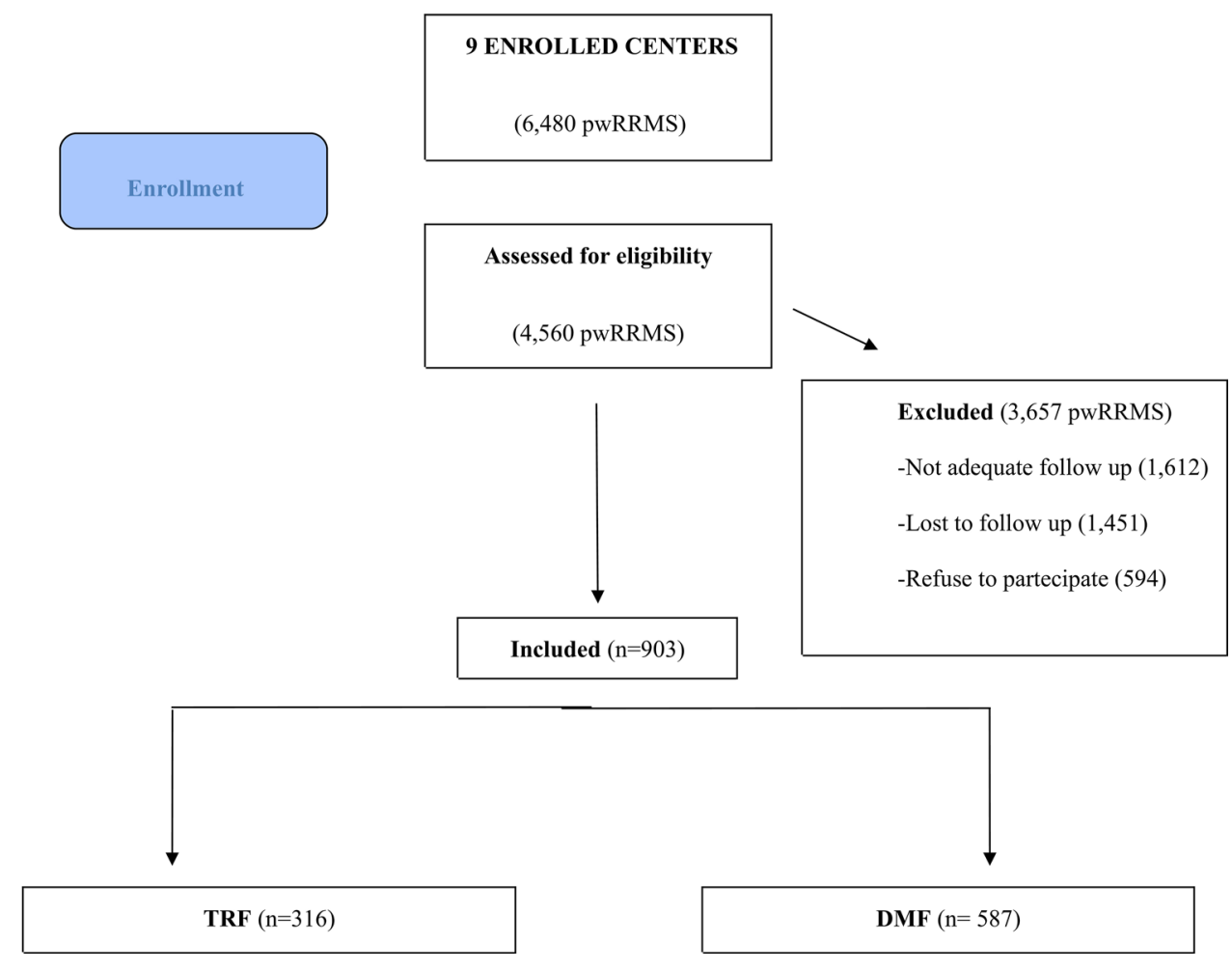

\begin{tabular}{llll}
\hline & TRF & DMF & $p$ value \\
\hline Variable & 316 & 587 & \\
Age & $46.3 \pm 10.3$ & $38.6 \pm 10.9$ & $<0.001$ \\
Gender & & & \\
Female, $n(\%)$ & $199(64)$ & $416(71)$ & $<0.05^{*}$ \\
Male, $n(\%)$ & $117(36)$ & $171(29)$ & \\
Disease duration (months) & $115.2 \pm 86.4$ & $103.2 \pm 82.8$ & $<0.05$ \\
Follow up duration (months) & $13.3 \pm 5.9$ & $13.8 \pm 6$ & $\mathrm{~ns}$ \\
Relapses 1 year before switch & $0.6 \pm 0.7$ & $0.7 \pm 0.8$ & $\mathrm{~ns}$ \\
EDSS 1 year before switch, median (IQR) & $2.0(1.5-3.5)$ & $1.5(1.0-3.0)$ & $<0.001$ \\
MRI Gd + 1 year before switch & $0.4 \pm 1.2$ & $0.5 \pm 1.0$ & $\mathrm{~ns}$ \\
Naive to treatment, number (\%) & $70(22.2)$ & $168(28.6)$ & $<0.05^{*}$ \\
Switchers, number (\%) & $246(77.8)$ & $419(71.4)$ & $<0.05^{*}$ \\
Tolerability & $162(65.8)$ & $294(70.2)$ & $\mathrm{ns}$ \\
Lack of efficacy & $84(34.2)$ & $125(29.8)$ & $\mathrm{ns}$ \\
Number of switches pre-treatment & $1.4 \pm 1.2$ & $1.5 \pm 1.2$ & \\
\hline
\end{tabular}

Results are expressed as mean $\pm \mathrm{sd}$ when otherwise specified

$D M F$ dimethyl fumarate, EDSS Expanded Disability Status Scale, $G d+$ gadolinium, MRI magnetic resonance imaging, $T R F=$ teriflunomide

*Via the Chi square test
Out of them, 26 pwRRMS (16 within 12 months, and 10 within 24 months) were in TRF group and 55 pwRRMS (30 within 12 months, and 25 within 24 months) were in DMF group.
The most frequent reason of discontinuation was a lack of effectiveness (in terms of experiencing a new clinical relapse or a new enhancing lesion at brain MRI) in 61 pwRRMS (19 on TRF and 42 on DMF with a mean time of $10.5 \pm 5$ 
and $13.3 \pm 3.5$, not statistically significant). AEs lead to discontinuation in 20 pwRRMS ( 7 on TRF and 13 on DMF, with a mean time of $11.9 \pm 5$ and $12.6 \pm 4.2$, not statistically significant). The most frequent AEs were gastrointestinal disturbances in both cohorts.

Kaplan-Meier estimates for the time to treatment discontinuation according to the DMTs are shown in Fig. 2; no differences exist between the two groups ( $p$ for log-rank test $=0.906$ ).
The analysis of predictors with Cox regression model showed differences between the two groups ( $p$ for $\log$ rank test $=0.007)$; in detail the male gender [HR 2.21 $(1.00-4.90) ; p=0.01]$ and the number of previous switches [HR $1.47(1.16-1.86) ; p=0.01]$ was associated with higher hazard of discontinuation in the DMF group (Fig. 3).

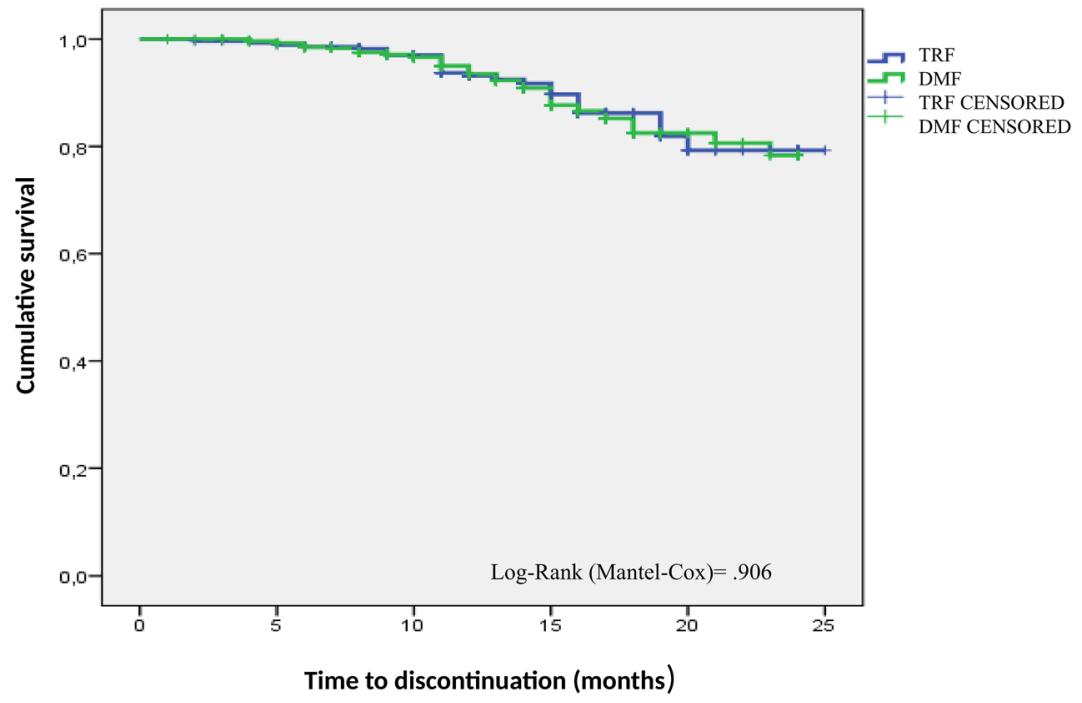

\begin{tabular}{|l|r|r|c|r|}
\hline & \multicolumn{3}{|c|}{} \\
\hline Treatment & N total & N. of & \multicolumn{2}{|c|}{ Censored } \\
\cline { 4 - 6 } & & events & N & \multicolumn{1}{c|}{ Ratio } \\
\hline TRF & 316 & 26 & 290 & $91.8 \%$ \\
\hline DMF & 587 & 55 & 532 & $90.6 \%$ \\
\hline Global & 903 & 81 & 822 & $91 \%$ \\
\hline
\end{tabular}

\begin{tabular}{|l|r|r|r|r|}
\hline \multicolumn{1}{|l}{ Treatment } & MEAN & \multicolumn{1}{|c|}{ Std. } & \multicolumn{2}{|c|}{ CI 95\% } \\
\cline { 3 - 5 } & & Error & Lower Limit & Upper Limit \\
\hline TNF & 22.8 & .403 & 22.1 & 23.6 \\
\hline DMF & 22.1 & .248 & 21.6 & 22.5 \\
\hline Global & 22.8 & .233 & 22.4 & 23.3 \\
\hline
\end{tabular}

Fig. 2 Time to discontinuation therapy between the two groups. $D M F$ dimethyl fumarate, $T R F$ teriflunomide

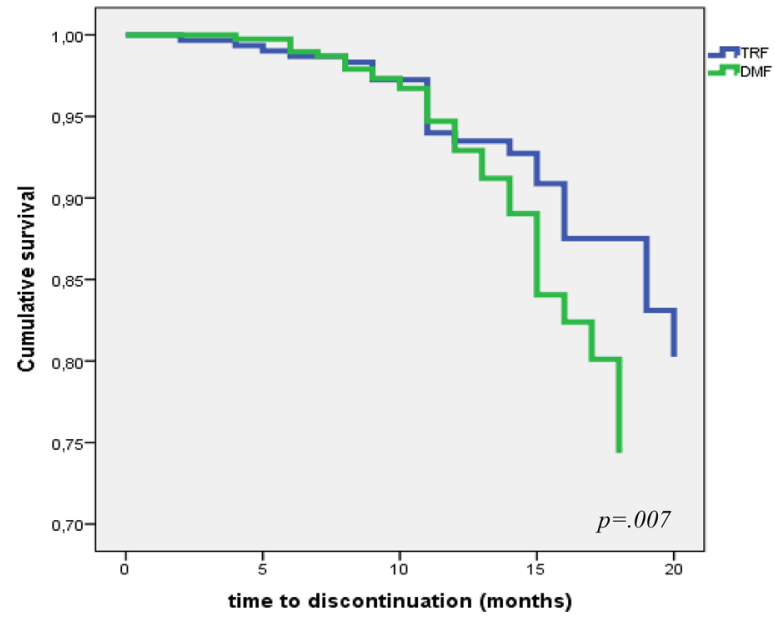

\begin{tabular}{lccc} 
& HR & CI 95\% & $p$ \\
\hline SEX & $\mathbf{2 . 2 1}$ & $\mathbf{1 . 0 0 - 4 . 9 0}$ & $\mathbf{. 0 5}$ \\
\hline CONDITIONAL DISEASE DURATION /AGE & 1.25 & $1.90-1.74$ & .17 \\
\hline RELAPSE 1 Y BEFORE TREATMENT & .68 & $.43-1.07$ & .09 \\
\hline EDSS 1 Y BEFORE TREATMENT & .92 & $.73-1.68$ & .51 \\
\hline Gd+ T1 MRI lesions 1Y before treatment & 1.22 & $.96-1.54$ & .88 \\
\hline NAIVE/SWITCHERS & 2.25 & $.72-6.94$ & .16 \\
\hline $\begin{array}{l}\text { N. SWITCHES BEFORE STARTING } \\
\text { TREATMENT }\end{array}$ & $\mathbf{1 . 5 5}$ & $\mathbf{1 . 1 1 - 2 . 1 7}$ & $\mathbf{0 . 0 1}$ \\
\hline
\end{tabular}

Fig. 3 Cox regression model for time to discontinuation among the two groups. DMF dimethyl fumarate, EDSS Expanded Disability Status Scale; $G d+$ gadolinium, $M R I$ magnetic resonance imaging, $T R F$ teriflunomide, $Y$ year 


\section{Discussion}

In our study, TRF and DMF showed similar discontinuation rates during the observation period. For every cohort, about $9 \%$ of pwRRMS withdrew the therapy. Such values are lower than those reported in the registrative trials [16-19]. Male gender and previous number of switches were the strongest predictors of therapy discontinuation in DMF group. To the best of our knowledge, this is the first study comparing the discontinuation rates between TRF and DMF in an Italian MS setting at 12 and 24 months.

A recent Italian retrospective study, which compared the 12-month discontinuation rates in pwRRMS on oral DMTs (fingolimod, TRF and DMF), found a lower discontinuation rate in people on fingolimod, when compared to DMF and TRF [7]. For those starting DMF, the 12-month discontinuation rate was $21.9 \%$ (out of 114 pwRRMS); for those on TRF was $23.6 \%$ (out of 64 pwRRMS). In another geography (US), a retrospective database study (based only on administrative claims data) compared the 12-month discontinuation rates among pwRRMS who initiated the oral DMTs. Regarding TRF and DMF, the authors found no differences of values (49.7\% vs 55.9\%, respectively) [6]. A recent Italian multicentre study reported a discontinuation rate of about $20 \%$ out of 70 patients treated with DMF at 24 months [14].

We found lower values than reported in the literature so far, and that could be linked to several factors. First, differences in the prevention and management of AEs among MS centers could help to explain such discrepancy [20]. For instance, some MS specialists suggest food-based management strategies (high fat, high protein, low starch, etc.) to reduce the impact of gastro-enteric AEs with DMF therapy [21]. Obviously, a lower discontinuation rate than expected could be related to an underestimation of AEs or an error in the collection of any AE [22].

Interestingly, we found that male gender (unmodifiable factor) and the previous number of switches (modifiable factor) were the strongest predictors of therapy discontinuation for patients on DMF.

Such data deserve attention and further studies are needed to better clarify the factors associated with risk of discontinuation of DMTs for pwRRMS in a real word setting.

In the clinical practice, pwRRMS with a history of therapeutic switches have experienced therapeutic failures and/or AEs. Such background could be due to the great subject variability in MS disease course and we might speculate that could be something similar to an individual predisposition to keep such status. About the gender, we could speculate a different profile of persistence to therapy (e.g. phycological reaction to the MS diagnosis, to the start of therapy, etc.); we need more real-life studies that should be designed to explore such patients reported outcomes.
Some limits of our study deserve attention. The nonrandomized design did not weigh the selection bias, as the medical decision to assign a pwRRMS to a specific drug rather than another. Nor did the other potential differences in baseline pwRRMS characteristics unless normally included in multivariate analysis.

More randomized long-term studies are needed to confirm our findings.

Acknowledgements Authors are grateful to Gregory Scott, $\mathrm{PhD}$ for language editing.

\section{Compliance with ethical standards}

Ethical standards The study has been approved by the local ethics committee (Catania 1) and has been performed in accordance with the ethical standards laid down in the 1964 Declaration of Helsinki and its later amendments. All persons gave their informed consent prior to their inclusion in the study. Details that might disclose the identity of the subjects under study have been omitted.

Conflicts of interest DE received personal fees by Biogen and Sanofi. $\mathrm{He}$ also received travel fundings from Bayer Biogen and Merck. ZA received travel fundings from Bayer-Schering and Sanofi Genzyme outside of the submitted work. BG received personal fees by Biogen and Sanofi. She also received travel fundings from Bayer Biogen and Merck. SM has nothing to disclose. CG received personal fees by Biogen and Sanofi. She also received travel fundings from Bayer Biogen and Merck. GA received personal fees by Biogen and Sanofi. He also received travel fundings from Bayer Biogen and Merck. SG received personal fees for speaking activities by Bayer Biogen Merck Novartis and Teva. BM received personal fees by Biogen and Sanofi. She also received travel fundings from Bayer Biogen and Merck. VP received personal fees by Biogen and Sanofi, Novartis and Roche. She also received travel fundings from Bayer Biogen and Merck. BRB served on the advisory board for Almirall and received grant for congresses participation by Sanofi and Merck. DR reports no disclosures. GLME served on the advisory board for Bayer, Biogen Celgene, Merck, Novartis, Roche, Sanofi and Teva. He also received personal fees for speaking activities at congresses or sponsored symposia. PC served on the advisory board for Bayer, Biogen Celgene, Merck, Novartis, Roche, Sanofi and Teva. He also received personal fees for speaking activities at congresses or sponsored symposia. TG served on the advisory board for Bayer, Biogen Celgene, Merck, Novartis, Roche, Sanofi, Teva and Almirall. He also received personal fees for speaking activities at congresses or sponsored symposia. ZM served on the advisory board for Bayer, Biogen Celgene, Merck, Novartis, Roche, Sanofi, Teva and Almirall. He also received personal fees for speaking activities at congresses or sponsored symposia. PF served on the advisory board for Bayer, Biogen Celgene, Merck, Novartis. Roche, Sanofi, Teva and Almirall. He also received personal fees for speaking activities at congresses or sponsored symposia.

\section{References}

1. Reich DS, Lucchinetti CF, Calabresi PA (2018) Multiple Sclerosis. N Engl J Med 378(2):169-180

2. D'Amico E, Zanghi A, Leone C, Tumani H, Patti F (2016) Treatment-related progressive multifocal leukoencephalopathy in 
multiple sclerosis: a comprehensive review of current evidence and future needs. Drug Saf 39(12):1163-1174

3. D'Amico E, Leone C, Caserta C, Patti F (2015) Oral drugs in multiple sclerosis therapy: an overview and a critical appraisal. Expert Rev Neurother 15(7):803-824

4. D'Amico E, Patti F, Zanghi A, Zappia M (2016) A personalized approach in progressive multiple sclerosis: the current status of disease modifying therapies (DMTs) and future perspectives. Int J Mol Sci 17(10):1725

5. Eichler HG, Abadie E, Breckenridge A, Flamion B, Gustafsson LL, Leufkens H et al (2011) Bridging the efficacy-effectiveness gap: a regulator's perspective on addressing variability of drug response. Nat Rev Drug Discov 10(7):495-506

6. Johnson KM, Zhou H, Lin F, Ko JJ, Herrera V (2017) Real-world adherence and persistence to oral disease-modifying therapies in multiple sclerosis patients over 1 year. J Manag Care Spec Pharm 23(8):844-852

7. Lattanzi S, Danni M, Taffi R, Cerqua R, Carlini G, Pulcini A et al (2017) Persistence to oral disease-modifying therapies in multiple sclerosis patients. J Neurol 264(11):2325-2329

8. Eriksson I, Cars T, Piehl F, Malmstrom RE, Wettermark B, von Euler M (2018) Persistence with dimethyl fumarate in relapsingremitting multiple sclerosis: a population-based cohort study. Eur J Clin Pharmacol 74(2):219-226

9. Ferraro D, Camera V, Baldi E, Vacchiano V, Curti E, Guareschi A et al (2018) First-line disease-modifying drugs in relapsingremitting multiple sclerosis: an Italian real-life multicenter study on persistence. Curr Med Res Opin 1:1-5

10. Sejbaek T, Nybo M, Petersen T, Illes Z (2018) Real-life persistence and tolerability with dimethyl fumarate. Mult Scler Relat Disord 24:42-46

11. Vollmer B, Ontaneda D, Bandyopadhyay A, Cohn S, Nair K, Sillau $\mathrm{S}$ et al (2018) Discontinuation and comparative effectiveness of dimethyl fumarate and fingolimod in 2 centers. Neurol Clin Pract 8(4):292-301

12. Mallucci G, Annovazzi P, Miante S, Torri-Clerici V, Matta M, La Gioia S et al (2018) Two-year real-life efficacy, tolerability and safety of dimethyl fumarate in an Italian multicentre study. $\mathrm{J}$ Neurol 265(8):1850-1859

13. Polman CH, Reingold SC, Banwell B, Clanet M, Cohen JA, Filippi $M$ et al (2011) Diagnostic criteria for multiple sclerosis: 2010 revisions to the McDonald criteria. Ann Neurol 69(2):292-302

14. D'Amico E, Zanghì A, Callari G, Borriello G, Gallo A, Graziano $\mathrm{G}$ et al (2018) Comparable efficacy and safety of dimethyl fumarate and teriflunomide treatment in relapsing-remitting multiple sclerosis: an Italian real-word multicenter experience. Ther Adv Neurol Disord 11:1756286418796404

15. https://www.ema.europa.eu/...guideline/guideline-good-pharm acovigilance-practices-gv. Accessed Oct 2018

16. Confavreux C, O'Connor P, Comi G, Freedman MS, Miller AE, Olsson TP et al (2014) Oral teriflunomide for patients with relapsing multiple sclerosis (TOWER): a randomised, double-blind, placebo-controlled, phase 3 trial. Lancet Neurol 13(3):247-256

17. Fox RJ, Miller DH, Phillips JT, Hutchinson M, Havrdova E, Kita $M$ et al (2012) Placebo-controlled phase 3 study of oral BG-12 or glatiramer in multiple sclerosis. N Engl J Med 367(12):1087-1097

18. Gold R, Kappos L, Arnold DL, Bar-Or A, Giovannoni G, Selmaj $\mathrm{K}$ et al (2012) Placebo-controlled phase 3 study of oral BG-12 for relapsing multiple sclerosis. N Engl J Med 367(12):1098-1107

19. O'Connor P, Comi G, Freedman MS, Miller AE, Kappos L, Bouchard JP et al (2016) Long-term safety and efficacy of teriflunomide: nine-year follow-up of the randomized TEMSO study. Neurology 86(10):920-930

20. Lugaresi A, Rottoli MR, Patti F (2014) Fostering adherence to injectable disease-modifying therapies in multiple sclerosis. Expert Rev Neurother 14(9):1029-1042

21. Theodore Phillips J, Erwin AA, Agrella S, Kremenchutzky M, Kramer JF, Darkes MJ et al (2015) Consensus management of gastrointestinal events associated with delayed-release dimethyl fumarate: a Delphi study. Neurol Ther 4(2):137-146

22. Ziemssen T, Kern R, Thomas K (2016) Multiple sclerosis: clinical profiling and data collection as prerequisite for personalized medicine approach. BMC Neurol 16(1):124 